\title{
High-Acoustic-Impedance Tantalum Oxide Layers for Insulating Acoustic Reflectors
}

\author{
Jose Capilla, Jimena Olivares, Marta Clement, Jesús Sangrador, Enrique Iborra, and Arnaud Devos
}

\begin{abstract}
This work describes the assessment of the acoustic properties of sputtered tantalum oxide films intended for use as high-impedance films of acoustic reflectors for solidly mounted resonators operating in the gigahertz frequency range. The films are grown by sputtering a metallic tantalum target under different oxygen and argon gas mixtures, total pressures, pulsed dc powers, and substrate biases. The structural properties of the films are assessed through infrared absorption spectroscopy and X-ray diffraction measurements. Their acoustic impedance is assessed by deriving the mass density from $\mathrm{X}$-ray reflectometry measurements and the acoustic velocity from picosecond acoustic spectroscopy and the analysis of the frequency response of the test resonators.
\end{abstract}

\section{INTRODUCTION}

$\mathrm{B}$ ULK acoustic wave resonator technology is a recent solution for low-cost, high-performance bandpass filters for digital wireless communications, such as wireless networking, cellular phones, or global positioning systems. These filters are composed of several resonators electrically connected to achieve the desired filter characteristics. To obtain well-performing devices, a good acoustic isolation of the resonators is required to avoid energy losses through the substrate that reduce their quality factor $(Q)$. There are two typical methods to achieve a good acoustic isolation. The first method consists of creating an air gap between the resonator and the substrate; because the acoustic radiation to the air is minimal, energy losses can only take place through the supports. These structures, called film bulk acoustic resonators (FBAR), have some drawbacks; they require complex fabrication technology and exhibit a too-high thermal isolation that limits power handling. The alternative method is the use of acoustic reflectors between the resonators and the substrate; the resulting structures are called solidly mounted resonators (SMR) [1]. Bragg mirrors are typical acoustic reflectors formed by alternating quarter-wavelength-thick layers ( $\lambda / 4$-layers) with low and high acoustic impedance [2].

Manuscript received August 15, 2011; accepted December 12, 2011. This work has been supported by the Ministerio de Ciencia e Innovación of Spain through project MAT2010-18933, by the Comunidad de Madrid and the UPM through the IV PRICIT program and by the European Union through the European Regional Development Fund (FEDER).

J. Capilla, J. Olivares, M. Clement, J. Sangrador and E. Iborra are with the Grupo de Microsistemas y Materiales Electrónicos del Centro de Materiales y Dispositivos Avanzados para las Tecnologías de la Información y Comunicaciones (GMME-CEMDATIC), Universidad Politécnica de Madrid, Madrid, Spain (e-mail: mclement@etsit.upm.es).

A. Devos is with the Institut d'Électronique, Microélectronique et Nanotechnologie, Institut Supérieur d'Électronique et du Numérique, UMR Centre National de la Recherche Scientifique 8520, Lille, France.

DOI: http://dx.doi.org/10.1109/TUFFC.2012.2205
The acoustic isolation achieved (reflection coefficient) increases with the number of layers and with the mismatch between the acoustic impedances of two consecutive layers. Considering that the acoustic impedance of a given material is the product of the longitudinal sound velocity and the mass density, these two material properties must be perfectly known to set the thickness of the films to $\lambda / 4$ to get the targeted resonant frequency and to fix the number of layers required for a specified mirror reflectance. Table I summarizes the experimental values of such material properties for the most commonly used acoustic mirror constituents.

Silicon dioxide $\left(\mathrm{SiO}_{2}\right)$ is commonly used as a lowacoustic-impedance material, because it is easy to process and fully compatible with standard silicon technologies [3]. Other low-impedance materials are $\mathrm{Al}$ [4] and $\mathrm{SiOC}$ [5], although they are less frequently used because of the metallic character of $\mathrm{Al}$ and the elevated acoustic losses of SiOC. On the other hand, materials with high acoustic impedance are typically high-density metals exhibiting, additionally, a sufficient sound velocity, such as Ir [6], Mo [7], or W [8]. If these high-impedance layers are replaced by insulating films, the fabrication process of BAW filters can be significantly simplified, because there is no need to define the continuous metallic layers that short circuit the different resonators built on top of the same acoustic mirrors. Achieving insulating Bragg mirrors thus requires high-acoustic-impedance insulating layers, which are, unfortunately, not easily available. So far, insulating acoustic mirrors have been obtained by combining $\mathrm{SiO}_{2}$ or $\mathrm{SiOC}$ layers with $\mathrm{AlN}$ [9], $\mathrm{Si}_{3} \mathrm{~N}_{4}$ [5], or $\mathrm{Ta}_{2} \mathrm{O}_{5}$ [10], [11], although the acoustic properties of this last material have not been extensively characterized. However, none of these three materials exhibits really high acoustic impedances, compared with that offered by high-density metallic layers. The considerably high acoustic velocity of AIN combined with a low mass density (see table I) provides a moderate acoustic impedance. On the other hand, $\mathrm{Ta}_{2} \mathrm{O}_{5}$ films offer acoustic impedance similar to ANN by virtue of its high mass density, although the sound velocity appears to be considerably lower.

In this work, we have characterized the acoustic properties of $\mathrm{TaO}_{x}$ (with $x$ close to 2.5 ) films to investigate whether these layers, in combination with $\mathrm{SiO}_{2}$ films, may provide better acoustic isolation than $\mathrm{SiO}_{2} / \mathrm{AlN}$-based Bragg mirrors. The structure, mass density, and sound velocity of sputtered $\mathrm{TaO}_{x}$ films have been assessed by different techniques, which include the fabrication and characterization of specific devices for the evaluation of its sound velocity. 
Table I. Acoustic Impedance of Metallic and Insulating Materials.

\begin{tabular}{lccc}
\hline Material & $\begin{array}{c}\text { Mass density } \\
\left(\mathrm{kg} \cdot \mathrm{m}^{-3}\right)\end{array}$ & $\begin{array}{c}\text { Sound velocity } \\
\left(\mathrm{m} \cdot \mathrm{s}^{-1}\right)\end{array}$ & $\begin{array}{c}\text { Acoustic impedance } \\
\left(\mathrm{kg} \cdot \mathrm{m}^{-2} \cdot \mathrm{s}^{-1}\right)\end{array}$ \\
\hline $\mathrm{SiOC}$ & 1500 & 2400 & $3.6 \times 10^{6}$ \\
$\mathrm{Al}$ & 2700 & 5100 & $11.2 \times 10^{6}$ \\
$\mathrm{SiO}_{2}$ & 2200 & 6000 & $13.2 \times 10^{6}$ \\
$\mathrm{Si}_{3} \mathrm{~N}_{4}$ & 2200 & 9900 & $21.8 \times 10^{6}$ \\
$\mathrm{AlN}$ & 3300 & 11000 & $36.3 \times 10^{6}$ \\
$\mathrm{Mo}$ & 10300 & 6190 & $63.8 \times 10^{6}$ \\
$\mathrm{~W}$ & 19290 & 5180 & $99.9 \times 10^{6}$ \\
$\mathrm{Ir}$ & 22500 & 5300 & $119.2 \times 10^{6}$ \\
\hline
\end{tabular}

\section{EXPERIMENT}

\section{A. Sputtering Process}

$\mathrm{TaO}_{x}$ films were deposited in a Leybold Z-550 system (Leybold AG, Köln, Germany) by pulsed-de magnetron sputtering of a 150 -mm-diameter Ta target in $\mathrm{Ar} / \mathrm{O}_{2}$ admixtures. A pulsed-dc power of $50 \mathrm{kHz}$ and duty cycle $75 \%$ was applied to the target. Up to 150 films were deposited under different conditions by varying the total pulsed-dc power between 500 and $1200 \mathrm{~W}$, the total pressure in the chamber between 1.5 and $3.3 \mathrm{mTorr}$, and the percentage of $\mathrm{O}_{2}$ in the gas between $30 \%$ and $100 \%$. An RF source was used to polarize the substrate between $-50 \mathrm{~V}$ (with no RF power applied to it) and $-150 \mathrm{~V}$. The substrates were not intentionally heated before or during deposition.

The sputter process of a Ta metallic target in $\mathrm{Ar} / \mathrm{O}_{2}$ atmospheres shows a transitory behavior resulting from the oxidation of the target. When bombarding a clean Ta metallic surface at constant power with a gas containing a certain percentage of oxygen (less than 100\%), the time to stabilize the plasma impedance can reach several hours. To guarantee that the target had reached a stationary state before starting deposition, the target was first preconditioned at the deposition power in pure oxygen atmosphere; the voltage of the cathode attained a constant value after around $15 \mathrm{~min}$, when the plasma impedance reached the steady state. The selected gas mixture was then fed into the chamber at the chosen pressure and the voltage of the cathode controlled until the new steady state was attained (15 to $30 \mathrm{~min}$ ). At this moment, the shutter was opened for film deposition for the desired time. Subsequent depositions under identical conditions required only a few minutes for stabilization. Every time the deposition conditions were modified, the complete conditioning process was restarted. The $\mathrm{TaO}_{x}$ films were deposited on (100) silicon wafers for IR transmission measurements and on acoustic reflectors (described below) built on top of silicon substrates for the assessment of the sound velocity through the frequency response of test SMRs.

\section{B. Film Characterization}

The structure and morphology of the $150 \mathrm{TaO}_{x}$ films were evaluated by infrared transmission measurements carried out with a Nicolet 5-PC Fourier transform infrared (FTIR) spec- trophotometer (Thermo Scientific, Waltham, MA), which provided a straightforward assessment of the chemical bonding configuration of the films. We used non-polarized light at normal incidence over the 400 to $4000 \mathrm{~cm}^{-1}$ range with a spectral resolution of $4 \mathrm{~cm}^{-1}$. The absorption bands corresponding to the Si substrate were eliminated by subtracting the absorbance spectrum measured in bare Si. X-ray diffraction patterns of 20 representative films were measured in conventional Bragg-Brentano geometry in a Supratech XPert MRD diffractometer (Panalytical, Almelo, The Netherlands) between $2 \theta=10^{\circ}$ and $2 \theta=80^{\circ}$.

The assessment of the acoustic properties of $\mathrm{TaO}_{x}$ films requires very precise measurements of their mass density, acoustic velocity, and thickness. The thickness of the $\mathrm{TaO}_{x}$ films and that of the other films involved in test devices were measured with a Dektak 150 profilometer (Veeco Instruments, Plainview, NY). The density of the $\mathrm{TaO}_{x}$ layers was assessed by X-ray reflectometry (XRR) measurements using the same diffractometer operated at grazing incidence between $\theta=0.05^{\circ}$ and $\theta=2.5^{\circ}$. Finally, the longitudinal sound velocity was assessed using two different techniques. First, the sound velocity was measured in different test structures by the picosecond ultrasonic technique [12]. A second assessment was deduced from the frequency response of the electrical impedance of specific SMR test devices built on top of acoustic reflectors containing the $\mathrm{TaO}_{x}$ layers under study; this was measured with an Agilent PNA N5230A network analyzer (Agilent Technologies Inc., Santa Clara, CA). $\mathrm{TaO}_{x}$ films with thickness ranging between 150 and $1000 \mathrm{~nm}$ were inserted between a well-characterized acoustic reflector, composed of four alternating porous- $\mathrm{SiO}_{2}[13]$ and Mo films, and a Mo/AlN/Mo resonator, as shown in Fig. 1. The excitation of the resonator induced a $\lambda / 2$ resonance in the $\mathrm{TaO}_{x}$ film. The fitting of the experimental data with Mason's model allowed derivation of the longitudinal acoustic velocity, provided that the material properties and the thickness of all the layers involved in the device were well known.

\section{REsults AND DISCUSsION}

\section{A. Structure and Composition of Sputtered Tantalum Oxides}

Fig. 2 shows the IR transmission spectra of three $\mathrm{TaO}_{x}$ films of different thickness. The bands at 950 and $650 \mathrm{~cm}^{-1}$ 


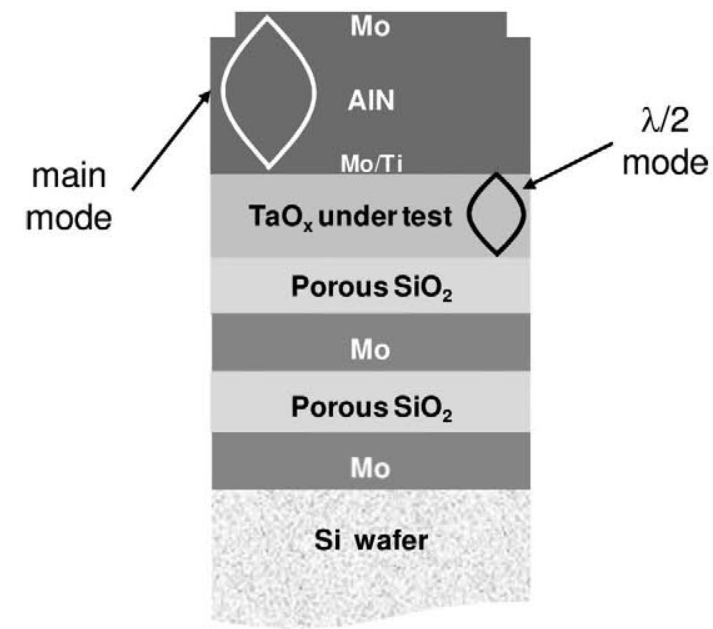

Fig. 1. Sketch of the test devices used for assessing the sound velocity in sputtered $\mathrm{TaO}_{x}$ films. The main resonant mode in the piezoelectric stack is represented in white, and the $\lambda / 2$ mode in the $\mathrm{TaO}_{x}$ film in black.

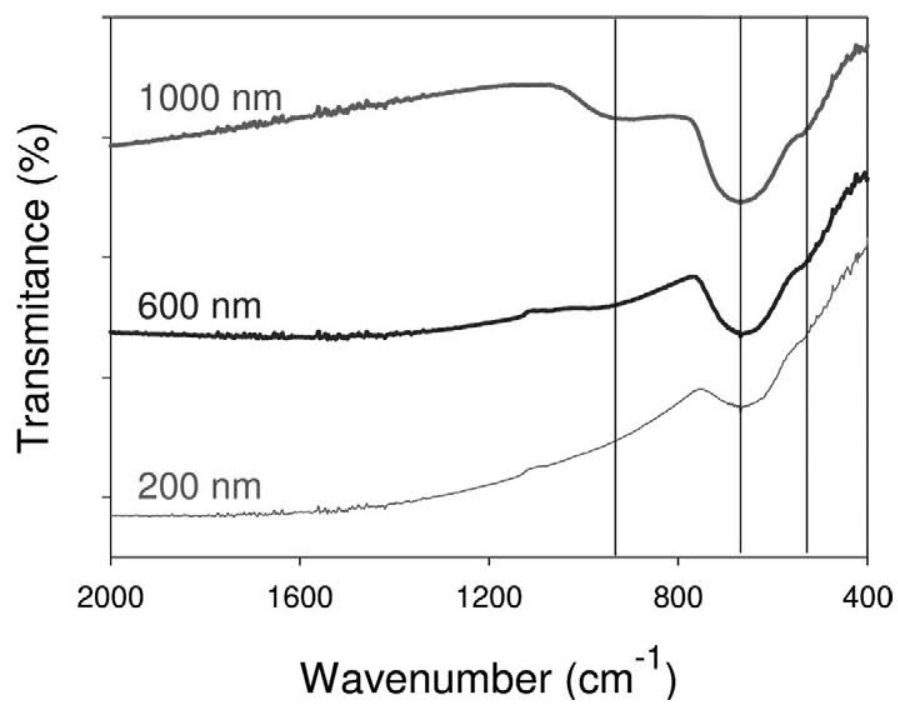

Fig. 2. Infrared transmision spectra between 2000 and $400 \mathrm{~cm}^{-1}$ of three $\mathrm{TaO}_{x}$ films of different thickness.

have been associated with the Ta-O-Ta stretching mode and with the $\mathrm{O} \equiv \mathrm{Ta}$ bond, respectively, of amorphous $\mathrm{TaO}_{x}$ films [14]. The main absorption band at $650 \mathrm{~cm}^{-1}$ has been reported to shift to lower wavenumbers (down to $510 \mathrm{~cm}^{-1}$ ) in polycrystalline films. In our spectra, this band appears as a shoulder in the $530 \mathrm{~cm}^{-1}$ region. The spectra suggest that our $\mathrm{TaO}_{x}$ films are mainly amorphous, but contain a certain amount of microcrystals. Considering the amplitude ratio of the bands at 530 and $650 \mathrm{~cm}^{-1}$ as a qualitative estimation of the amount of microcrystals embedded in the amorphous matrix, a slight increase is observed with the thickness of the films. However, in films of similar thickness, we have not observed any clear dependence between the amount of microcrystals and the deposition conditions used in this work.

$\mathrm{X}$-ray diffraction measurements confirm this behavior. Fig. 3 shows the XRD patterns of the three $\mathrm{TaO}_{x}$ films of different thickness analyzed by FTIR. It is important to

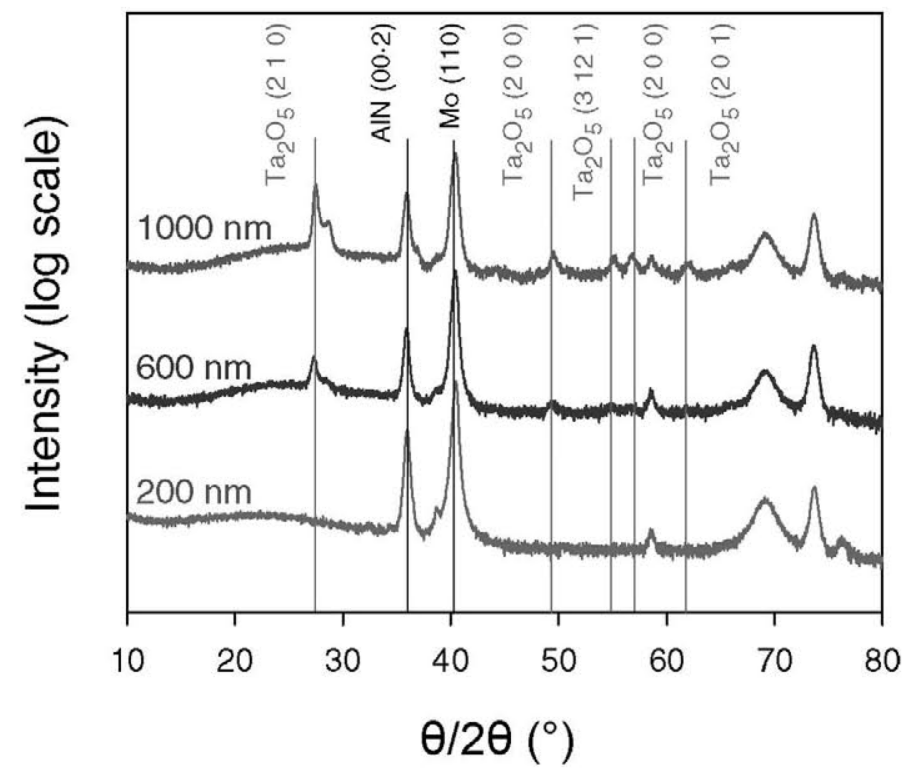

Fig. 3. X-ray diffraction patterns of $\mathrm{TaO}_{x}$ films of different thickness.

note that, among all the films deposited, only a few exhibited clear XRD peaks corresponding to the orthorhombic $\beta$-phase of $\mathrm{Ta}_{2} \mathrm{O}_{5}$, whereas most of the films deposited in similar conditions were clearly amorphous. Peaks at $2 \theta$ $=26^{\circ}, 52^{\circ}, 48^{\circ}, 56^{\circ}$, and $62^{\circ}$ corresponding to the (210), (200), (3121), (200), and (201) directions of the $\mathrm{Ta}_{2} \mathrm{O}_{5}$ orthorhombic $\beta$-phase appear in the patterns corresponding to the thicker films, whereas no traces of these peaks are visible in the thinner one, which indicates its amorphous character. Additional peaks corresponding to AlN and Mo are also observed, because the films are characterized after depositing the piezoelectric stack for electric characterization. The data suggest again that thicker films tend to contain some nanocrystals whose grain size, derived from the width of the $\mathrm{TaO}_{x}$ peaks, is around $10 \mathrm{~nm}$. However, we have not found any clear trend between the microcrystalline character of the samples (or any other film property) and the deposition conditions.

\section{B. Density Assessment}

The mass density $(\rho)$ of the $\mathrm{TaO}_{x}$ films was measured by XRR, which provides a direct assessment of $\rho$, independent of the thickness. X-rays impinging on smooth $\mathrm{TaO}_{x}$ surfaces at grazing incidence below a critical angle $\left(\theta_{c}\right)$ are totally reflected. When the incident angle exceeds $\theta_{\mathrm{c}}$, the X-rays penetrate into the film and the intensity of the reflected beam drops sharply. According to [15], $\rho$ can be obtained through the measured $\theta_{\mathrm{c}}$. The XRR measurements corresponding to the films exhibiting the greatest and the lowest density are shown in Fig. 4; the curves of all the remaining films lie between these two traces. The experimental data were fitted using the commercial software RCREFSimW (Innovations for High Performance Microelectronics, Frankfurt, Germany) which provided an accurate value of $\rho$. The values obtained were 8100 and $7300 \mathrm{~kg} \cdot \mathrm{m}^{-3}$, respectively, revealing that even the denser 


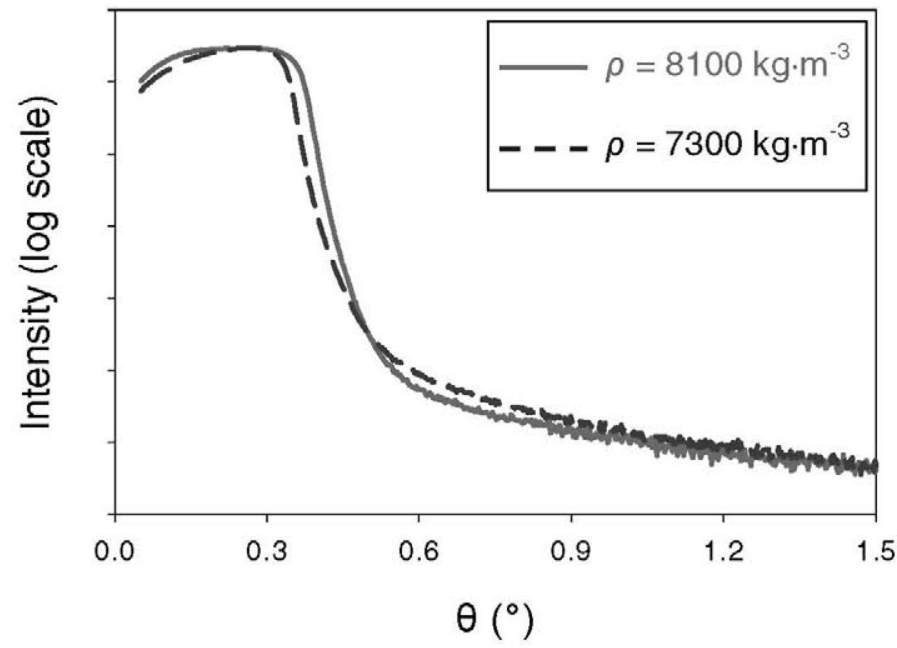

Fig. 4. X-ray reflection curves of two 200-nm-thick $\mathrm{TaO}_{x}$ films grown on acoustic reflectors. The solid line corresponds to the film of highest mass density and the dashed line corresponds to the film of lowest mass density.

films do not reach the nominal mass density of $\mathrm{Ta}_{2} \mathrm{O}_{5}$ $\left(8200 \mathrm{~kg} \cdot \mathrm{m}^{-3}\right)$. These low values of $\rho$ could be associated either with a lack of stoichiometry (films containing oxygen in excess) or, more likely, with a non-packed structure associated with the amorphous structure of most of the films. The high value of the refractive index (2.26) measured at a wavelength of $400 \mathrm{~nm}$ confirms this hypothesis.

\section{Sound Velocity Assessment}

1) Assessment Through Test SMRs: The first way to assess the longitudinal sound velocity in the $\mathrm{TaO}_{x}$ films consisted of the measurement of the $\lambda / 2$ resonant mode induced in these layers when they were placed in intimate contact with a piezoelectric resonator, as shown in Fig. 1. Taking into account that the wavelength of the stationary wave of this mode is ideally twice the thickness of the layer, the sound velocity $v_{\mathrm{s}}$ can be estimated, in a first approach, from the resonant frequency $f_{0}$ of the $\lambda_{\mathrm{TaO} x} / 2$ mode and the thickness $t$ of the layer through

$$
v_{\mathrm{s}}=f_{0} \times 2 \cdot t
$$

However, because the resonant frequencies of all of the modes also depend on the weight of the different layers involved in the test SMR, reliable values of the longitudinal sound velocity were only achieved if the whole experimental frequency response of the test resonator was fitted with Mason's physical model [16].

The variation with frequency of the modulus of the electrical impedance of two representative test SMRs containing $\mathrm{TaO}_{x}$ layers of different thickness is shown in Fig. 5. Two resonances appear in each spectrum at different frequencies. The low-frequency modes correspond to the main resonance in the AlN layer, whereas the high-frequency modes are associated with the $\lambda / 2$ mode in the

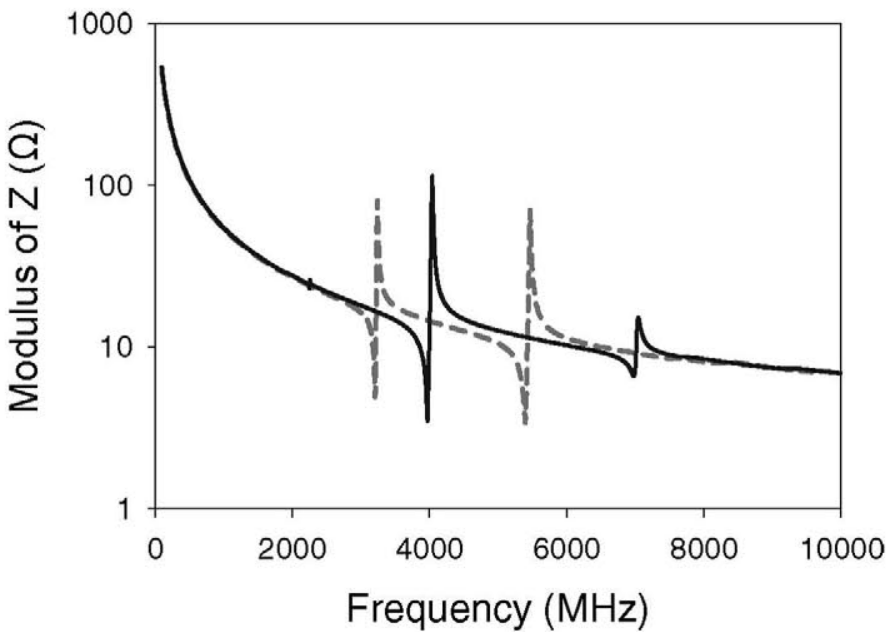

Fig. 5. Modulus of the impedance versus frequency of two otherwise identical test SMRs containing 180 -nm-thick (solid line) and 400-nm-thick (dashed line) $\mathrm{TaO}_{x}$ films.

TABle II. Material Constants for. Solidly Mounted Resonator. Materials.

\begin{tabular}{lcc}
\hline Material & $v_{\mathrm{s}}(\mathrm{m} / \mathrm{s})$ & $\rho\left(\mathrm{kg} \cdot \mathrm{m}^{-3}\right)$ \\
\hline $\mathrm{Mo}$ & 6290 & 10300 \\
$\mathrm{SiO}_{2}$ & 5350 & 1900 \\
$\mathrm{AlN}$ & 11000 & 3300 \\
\hline
\end{tabular}

$\mathrm{TaO}_{x}$ film. Fig. 5 shows that upon decreasing the thickness of the $\mathrm{TaO}_{x}$ film, the two main resonances shift to higher frequencies, not only because of the lower value of $\lambda / 2$ but also as a consequence of the reduction of the weight of this layer, which also affects the propagation of the wave in the whole structure.

Fitting the experimental data to Mason's model requires precise values of the material properties of all layers involved in the test structures and of all the dimensions of the devices (thickness of layers and areas of the resonators). The areas of the top electrodes, which defined the areas of the SMRs, were measured carefully with an optical microscope to avoid errors resulting from the undercutting during the electrode definition. The properties of the different materials used for fitting the experimental data are listed in Table II. Data for Mo and AIN were taken from the literature and those corresponding to porous $\mathrm{SiO}_{2}$ had been carefully characterized in a previous work [13]. All these properties have also been routinely tested in other devices that do not contain $\mathrm{TaO}_{x}$ films.

The fitting of the experimental data of Fig. 5 allowed deduction of the values of the longitudinal sound velocity of the different $\mathrm{TaO}_{x}$ layers. These are depicted as a function of the thickness of the $\mathrm{TaO}_{x}$ slab in Fig. 6 along with the value of the sound velocity deduced from the alternative technique described in Section III-C-2.

2) Assessment Through Picosecond Measurements: The aim of these experiments is to measure the time-of-flight $(\tau)$ of a longitudinal acoustic wave within the $\mathrm{TaO}_{x}$ films 


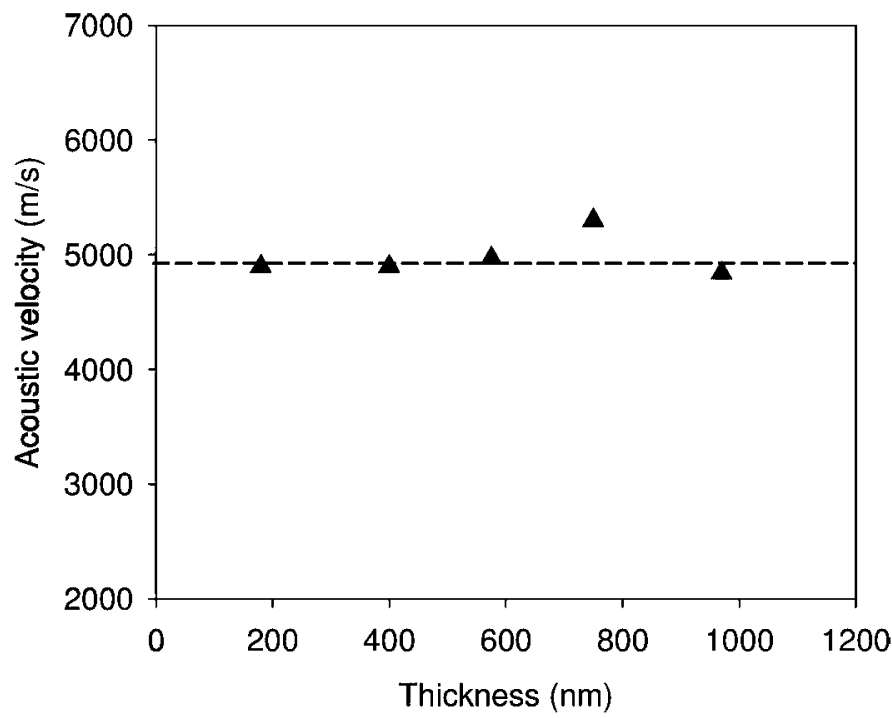

Fig. 6. Sound velocity of a set of $\mathrm{TaO}_{x}$ of different thicknesses grown under identical conditions obtained from BAW devices measurements (triangles). The line shows the mean value obtained by picosecond measurements.

deposited on top of the Bragg mirrors. If the thickness $d$ of the layer is known, the sound velocity is obtained by dividing $d$ by $\tau$. In this experiment, the acoustic wave is generated by means of a pump laser pulse that impinges the surface of the sample; a strong absorption takes place when the laser pulse reaches the first metallic layer it encounters, which produces a local heating of the metallic layer and hence a strain pulse that propagates toward the surface at the longitudinal velocity. The arrival of the acoustic pulse to the surface is detected by using a second laser beam, which allows measurement of the timeof-flight of the acoustic pulse in the layer under study [17]. In our samples, the laser pulse travels through two transparent media $\left(\mathrm{TaO}_{x}\right.$ and $\mathrm{SiO}_{2}$ films) until it reaches the surface of the first absorbing Mo layer of the acoustic reflector, where the longitudinal strain wave is generated. The acoustic pulse emitted from the buried Mo layer propagates toward the free surface through the transparent $\mathrm{SiO}_{2}$ and $\mathrm{TaO}_{x}$ bi-layer. The arrival of the pulse at the surface is detected as a reflectivity step which is due to the acoustic reflection at the free surface, as shown in Fig. 7. Such a reflectivity step is easily detected, with accuracy better than $2 \mathrm{ps}$ in any structure made of transparent/absorbing layers. A more detailed explanation can be found in [18].

The time delay $\tau_{\exp }$ deduced in our measurements includes the time-of-flight of the echo through both the $\mathrm{SiO}_{2}$ layer $\left(\tau_{\mathrm{SiO}_{2}}\right)$ and the $\mathrm{TaO}_{x}$ layer $\left(\tau_{\mathrm{TaO} x}\right)$, and hence can be written as:

$$
\tau_{\exp }=\tau_{\mathrm{Ta}_{2} \mathrm{O}_{5}}+\tau_{\mathrm{SiO}_{2}}=\frac{d_{\mathrm{Ta}_{2} \mathrm{O}_{5}}}{v_{\mathrm{Ta}_{2} \mathrm{O}_{5}}}+\tau_{\mathrm{SiO}_{2}} .
$$

To deduce the sound velocity in the $\mathrm{TaO}_{x}$ slab $\left(v_{\mathrm{Ta}, \mathrm{O} x}\right)$, we have studied the time delay at which the reflectivity step is observed for a set of $\mathrm{TaO}_{x}$ layers of different thicknesses

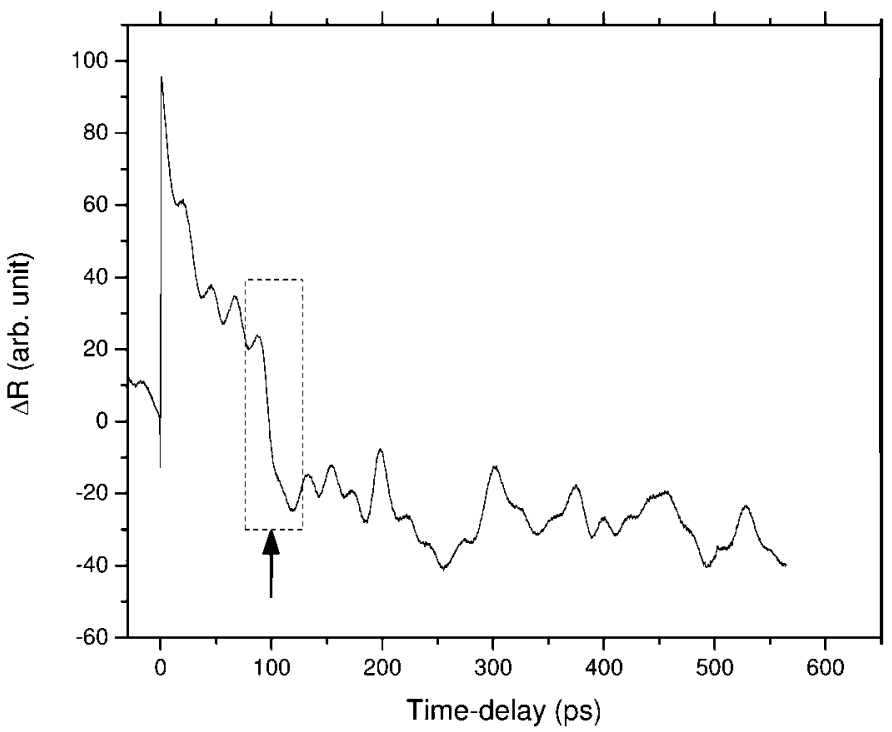

Fig. 7. Reflectivity signal as a function of time obtained in a 180-nmthick $\mathrm{TaO}_{x}$ layer grown on top of an acoustic reflector. The arrow close to 100 ps shows the sudden step resulting from the reflection of the acoustic pulse at the free surface, corresponding to a single way through the bilayer $\mathrm{SiO}_{2}$ and $\mathrm{TaO}_{x}$.

$\left(d_{\text {ТaО } x}\right)$ deposited on identical acoustic reflectors. Because the $\mathrm{SiO}_{2}$ layer is identical for each sample, by plotting the evolution of the time-delay as a function of the $\mathrm{TaO}_{x}$ thickness and fitting the data using a linear regression (see Fig. 8) we obtain the value of $v_{\mathrm{TaO} x}$ (inverse of the slope) and that of $\tau_{\mathrm{SiO} 2}$ (ordinate at the origin).

The mean value of the sound velocity obtained by this technique is $4900 \mathrm{~m} \cdot \mathrm{s}^{-1}$, which is in very good agreement with those achieved by the BAW resonator fitting method (see Fig. 6). The two methods suggest that the $750 \mathrm{~nm}-$ thick sample possesses a sound velocity $\left(5300 \mathrm{~m} \cdot \mathrm{s}^{-1}\right)$ significantly greater than the other samples, which indicates that there might be some influence of the growth conditions and the structure of the substrates on the properties of the $\mathrm{TaO}_{x}$ films, as suggested by XRD measurements. Indeed, other measurements of $v_{\mathrm{TaO} x}$ carried out in films grown on $\mathrm{Si}$ and Mo substrates reveal that $v_{\mathrm{TaO} x}$ tends to be slightly lower in films grown on $\mathrm{Si}$, whereas $v_{\mathrm{Ta}_{\mathrm{O}} \mathrm{x}}$ of films deposited on Mo layers reach greater values (around $5500 \mathrm{~m} \cdot \mathrm{s}^{-1}$ ).

\section{Acoustic Reflector Simulations}

To investigate whether these $\mathrm{TaO}_{x}$ films may contribute to optimize the performance of insulating acoustic reflectors, we have simulated the acoustic transmittance of mirrors composed of nine alternating layers of porous $\mathrm{SiO}_{2}$ and $\mathrm{TaO}_{x}$ films and compared it with that of conventional AlN-based mirrors. The simulations, shown in Fig. 9, suggest that even for the lowest values of the mass density and acoustic velocity, $\mathrm{TaO}_{x}$-based mirrors exhibit lower transmission coefficients in a wider frequency band than AlN-based reflectors. Additionally, the dispersion of the values of the mass density and acoustic velocity does not 


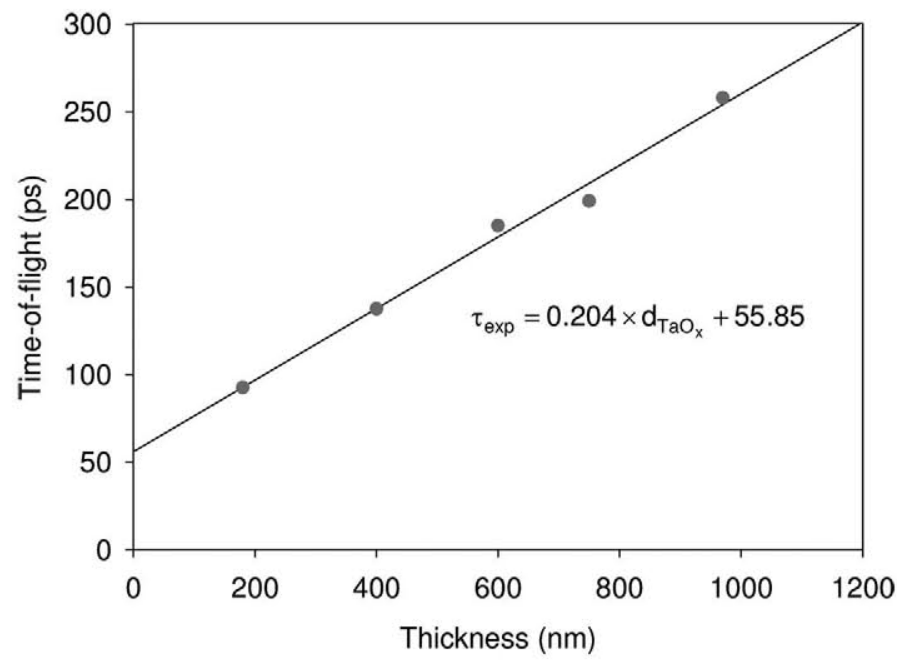

Fig. 8. Time of flight for several samples with different $\mathrm{TaO}_{x}$ thickness.

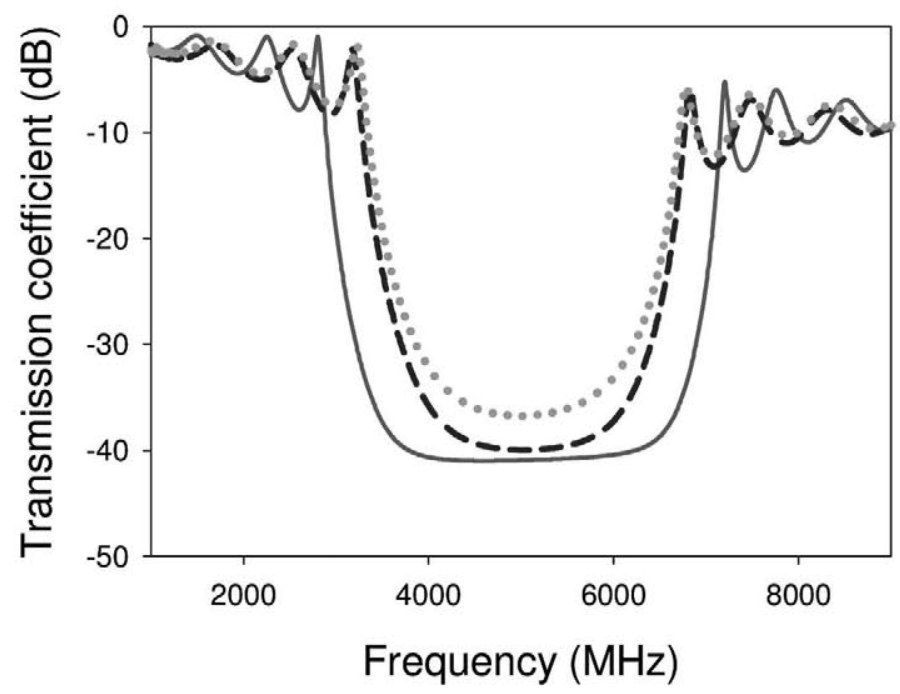

Fig. 9. Simulated transmission coefficient of acoustic reflectors composed of nine porous $\mathrm{SiO}_{2} / \mathrm{TaO}_{x}$ layers using the greatest values of $\rho$ and $v_{\mathrm{s}}$ (solid line) and the smallest values of $\rho$ and $v_{\mathrm{S}}$ (dashed line) compared with that of conventional $\mathrm{SiO}_{2} / \mathrm{AlN}$ reflectors (dotted line).

affect significantly the performance of the reflectors in the middle of the band.

Preliminary results on the performance of AIN-based SMRs using insulating $\mathrm{TaO}_{x} / \mathrm{SiO}_{2}$ acoustic reflectors yield quality factors up to 800 at $8 \mathrm{GHz}$.

\section{CONCLUSIONS}

In this paper we have assessed the acoustic properties of $\mathrm{TaO}_{x}$ films deposited at room temperature by reactive pulsed-dc sputtering. The sound velocity derived from picosecond measurements and the frequency response of BAW test devices depends slightly on the thickness of the films, which has been associated with their different structures. Thinner films appear to be amorphous, whereas films that are at least $600 \mathrm{~nm}$ thick tend to exhibit some nanocrystallites. The typical sound velocity in films with thickness below $400 \mathrm{~nm}$ is around $4900 \mathrm{~m} \cdot \mathrm{s}^{-1}$. The mass density of the $\mathrm{TaO}_{x}$ films was assessed by X-ray reflectometry; the average value is $7600 \mathrm{~kg} / \mathrm{m}^{3}$, around $93 \%$ of the nominal density of $\mathrm{Ta}_{2} \mathrm{O}_{5}$ films. The typical acoustic impedance of the films is $37.2 \times 10^{6} \mathrm{~kg} \cdot \mathrm{m}^{-2} \cdot \mathrm{s}^{-1}$, which is slightly greater than that of AlN. Combined with a low acoustic impedance material, such as porous $\mathrm{SiO}_{2}, \mathrm{TaO}_{x}$ films allow fabrication of all-dielectric acoustic reflectors with good performances for gigahertz-range filtering applications.

\section{REFERENCES}

[1] R. Ruby, "Review and comparison of bulk acoustic wave FBAR, SMR technology," in Proc. 2007 IEEE Int. Ultrasonics Symp., pp. 1029-10407.

[2] K. M. Lakin, K. T. McCarron, and R. E. Rose, "Solidly mounted resonators and filters," in Proc. 1995 IEEE Int. Ultrasonics Symp., pp. 905-908.

[3] S. Marksteiner, J. Kaitila, G. G. Fattinger, and R. Aigner, "Optimization of acoustic mirrors for solidly mounted BAW resonators," in Proc. 2005 IEEE Int. Ultrasonics Symp., vol. 1, pp 329-332.

[4] J. Enlund, D. Martin, V. Yantchev, and I. Katardjiev, "Solidly mounted thin film electro-acoustic resonator utilizing a conductive Bragg reflector," Sens. Actuators A, vol. 141, pp. 598-602, Feb. 2008.

[5] M. Clement, E. Iborra, J. Olivares, N. Rimmer, S. Giraud, S. Bila, and A. Reinhardt, "DCS Tx filters using AlN resonators with iridium electrodes," Trans. Ultrason. Ferroelectr. Freq. Control, vol. 57, no. 3, pp. 518-523, 2010.

[6] J. Olivares, E. Wegmann, M. Clement, J. Capilla, E. Tborra, and J. Sangrador, "Wide bandwidth Bragg mirrors for multi-band filter chips," in Proc. 2009 IEEE Int. Ultrasonics Symp., pp. 2119-2122.

[7] C.-J. Chung, Y.-C. Chen, C.-C. Cheng, and K.-S. Kao, "Fabrication and frequency response of solidly mounted resonators with $1 / 4 \lambda$ mode configuration," Thin Solid Films, vol. 516, pp. 5277-5281, Jun. 2008.

[8] R. Aigner, J. Kaitila, J. Ella, L. Elbrecht, W. Nessler, M. Handtmann, T.-R. Herzog, and S. Marksteiner, "Bulk-acoustic-wave filters: Performance optimization and volume manufacturing," in Proc. 2003 IEEE Int. MTT-S Digest, pp. 2001-2004.

[9] S. H. Kim, J. H. Kim, J. K. Lee, S. H. Lee, and K. H. Yoon, "Bragg reflector thin film resonator using aluminium nitride deposited by RF sputtering," in Proc. 2000 IEEE Microwave Conf. Asia-Pacific, pp. $1535-1538$.

[10] J.-W. Lobeek, R. Strijbos, A. Jansman, N. X. Li, A. B. Smolders, and N. Puisford, "High-Q BAW resonator on Pt/ $\mathrm{Ta}_{2} \mathrm{O}_{5} / \mathrm{SiO}_{2}$-based reflector stack," in Proc. 2007 IEEE International MTT-S Digest, pp. 2047-2050.

[11] H. P. Loebl, C. Metzmacher, R. F. Milsom, R. Mauczok, W. Brand, P. Lok, A. Tuinhout, and F. Vanhelmont, "Narrow band bulk acoustic wave filters," in Proc. 2004 IEEE Int. Ultrasonics Symp., pp. 411-415.

[12] P. Emery, G. Caruyer, R. Vélard, N. Casanova, P. Ancey, and A. Devos, "Picosecond ultrasonics: An original tool for physical characterization of Bragg reflectors in bulk acoustic wave resonators," in Proc. 2005 IEEE Int. Ultrasonics Symp., pp. 906-909.

[13] J. Olivares, E. Wegmann, J. Capilla, E. Iborra, M. Clement, L. Vergara, and R. Aigner, "Sputtered $\mathrm{SiO}_{2}$ as low acoustic impedance material for Bragg mirror fabrication in BAW resonators," IEEE Trans. Ultrason. Ferroelectr. Freq. Control, vol. 57, no. 1, pp. 23-29, Jan. 2010.

[14] A. P. Huang, S. L. Xu, M. K. Zhu, B. Wang, and H. Yan, "Crystallization control of sputtered $\mathrm{Ta}_{2} \mathrm{O}_{5}$ thin films by substrate bias," Appl. Phys. Lett., vol. 83, no. 16, pp. 3278-3280, Oct. 2003.

[15] C. Ferrari, A. Libassi, B. K. Tanner, V. Stolojan, J. Yuan, L. M. Brown, S. E. Rodil, B. Kleinsorge, and J. Robertson, "Density, sp" fraction, and cross-sectional structure of amorphous carbon films 
determined by X-ray reflectivity and electron energy-loss spectroscopy," Phys. Rev. B, vol. 62, no. 16, pp. 11089-11103, Oct. 2000.

[16] J. F. Rosenbaum, Bulk Acoustic Wave Theory and Devices, Boston, MA: Artech House, 1988.

[17] P. Emery, A. Devos, and P. Ancey, "Picosecond ultrasonics: The preferred tool for BAW characterization," in Proc. 2008 IEEE Int. Ultrasonics Symp., pp. 2205-2208.

[18] A. Devos, J.-F. Robillard, R. Côte, and P. Emery, "High laser-wavelength sensitivity of the picosecond ultrasonic response in transpar- ent thin films," Phys. Rev. B, vol. 74, no. 6, art. no. 064114, Aug. 2006. 